\title{
Surfaces
}

\section{LA TROISIÈME CONFÉRENCE SUR LE CYBERSPACE}

\section{Ollivier Dyens}

Volume 3, 1993

URI : https://id.erudit.org/iderudit/1065108ar

DOI : https://doi.org/10.7202/1065108ar

Aller au sommaire du numéro

Éditeur(s)

Les Presses de l’Université de Montréal

ISSN

1188-2492 (imprimé)

1200-5320 (numérique)

Découvrir la revue

Citer ce compte rendu

Dyens, O. (1993). Compte rendu de [LA TROISIÈME CONFÉRENCE SUR LE

CYBERSPACE]. Surfaces, 3. https://doi.org/10.7202/1065108ar d'utilisation que vous pouvez consulter en ligne.

https://apropos.erudit.org/fr/usagers/politique-dutilisation/ 


\title{
LA TROISIÈME CONFÉRENCE SUR LE CYBERSPACE
}

\author{
COMPTE RENDU D'UNE CONFÉRENCE
}

Ollivier Dyens

Compte-rendu de LA TROISIEME CONFÉRENCE SUR LE CYBERSPACE. Organisée par Michael Benedikt et par le Département d'architecture de l'Université du Texas à Austin, la conférence a eu lieu les 14 et 15 mai 1993.

Le cyberspace, cet espace informatique de la réalité virtuelle et de l'Internet, cristallisé par l'écrivain américain William Gibson, a connu depuis quelques années un essor remarquable. De véritables études existent maintenant sur le sujet, et ce phénomène possède aujourd'hui ses écrivains, ses penseurs et ses conférences.

Organisée, comme les deux précédentes, par Michael Benedikt et son équipe, la troisième conférence sur le cyberspace, qui s'est tenue à Austin les 14-15 mai de cette année, a permis d'accroître les fondations épistémologiques de cette nouvelle humanité. Et il est de plus en plus juste de parler ici d'humanité, puisque cette forme de communication qui n'était, il n'y a pas si longtemps, qu'une simple technologie, se dote aujourd'hui d'une structure phénoménologique importante. Le cyberspace, cet océan "habitable" de réseaux et de signes, devient rapidement un alter-réel complexe et infini.

Quelques lignes de pensée principales, pour la plupart construites justement autour de cette idée d'alter-réel, ont émergé lors de cette troisième conférence. La relation cyberspace/mythologie/oralité, par exemple, est une 
voie phénoménologique souvent empruntée. Alors que Walter Ong proposait, il y a de ça déjà quelques années, le concept de seconde oralité pour l'espace médiatique contemporain, il est intéressant de relever les nombreuses références (conscientes ou inconscientes) qui lui ont été faites au cours de la conférence. Soulignons en particulier la présentation de David Sewell intitulé:"The USENET Oracle". Cette présentation, qui tentait de souligner la difficulté inhérente à la notion d'auteur dans l'Internet, a aussi prouvé combien l'absence d'identification au texte (courante dans nombre de regroupements cyberspatiaux) attribue à l'Internet une structure méta-fictive, puisque existe, parallèlement, le sentiment de manipuler et d'être manipulé par la fiction. Bien sûr, la présence souvent sous-jacente d'un "narrateur" (contrôleur, modérateur, etc.) omniscient et omniprésent qui s'insère dans nombre de communications joue pour beaucoup, mais d'autres éléments proposent à leur tour une explication. Il arrive souvent que le cyberspace semble posséder une volonté qui lui est propre; volonté marquée par les exigences techniques, mais aussi typographiques et cognitives qu'impose cet espace-temps. Si, comme le souligne David Porush, il est possible de percevoir le texte littéraire comme un réseau intelligent, il n'est alors pas impossible de concevoir que l'océan de textes liquides et mouvants que constitue le cyberspace impose à l'utilisateur qui le navigue un véritable dialogue être vivant/machine.

La perception du cyberspace comme signe d'un méta-lieu dans lequel une symbiose vivant/non-vivant est concevable, semble constituer une pensée charnière, commune à nombre de chercheurs. Plusieurs conférenciers ont d'ailleurs noté l'émergence, dans cet espace, d'organisations (signes, langues, intelligences) qui sembleraient organiques (ou, pour être plus juste, cyborganiques). Ainsi Michael Benedikt, en introduisant ces deux jours de débats, a présenté le terme de Community of Worldmaking, concept qui sous-entend la présence de toute une série de créations articulées et organisées. Ces créations, partagées entre l'être vivant et la machine, suggèrent donc une présence androïde. Mais il s'agit ici d'une présence androïde différente, dont le point de convergence est la transformation de tout corps (physique, cognitif, électromagnétique, algorithmique) en matrice informationelle. Ce concept sera d'ailleurs repris par Marcos Novak qui le considère même comme la métaphore principale de l'expression artistique contemporaine. Pour Novak le tout peut d'ailleurs se résumer par le terme de Liquid Architectures (développé il y a déjà deux ans lors de la première conférence sur le cyberspace). Pour ce professeur de l'Université du Texas, le concept d'architecture liquide représente une "danse" de désincarnation, par laquelle le corps sans organe de l'utilisateur se distribue dans le monde cyberspatial (où il peut donc être "happé", intégré, assimilé à la présence machine).

D'autres conférenciers, en particulier Eliot Handelman (Cybersemiosis) et Mark Pesce (Holisthetic Medium), se lanceront à corps perdu dans l'exploration d'une organicité cyberspatiale. Pour ces deux conférenciers, la possibilité de se fondre cognitivement mais aussi physiquement dans ce méta-lieu est tout à fait réelle. D'ailleurs, bien que poursuivant des buts 
divergeants (Handelman considérant cette possibilité comme un plus, Pesce comme un moins), ces auteurs s'accordent sur les conséquences possibles de cette mutation: tous deux soulignent la probable apparition de pathologies (donc de transformations) causées par la "liquéfaction" cyberspatiale de l'être humain.

Enfin plusieurs chercheurs ont présenté des idées qui, bien que d'une parenté plus éloignée, suggèrent aussi la possibilité d'un rapprochement entre l'être humain et la machine. Parmi ces derniers mentionnons les présentations de Thomas Mical (The Stealth Landscape), et de Mark C. Taylor ( Rhizomic Folds of Interstating). Pour Mical, l'espace cyberspatial est un espace belliqueux et militaire, essentiellement caché et absent, construit sur le mode de l'observation répressive. Pour Mark Taylor, le cyberspace, qui représente la culture du simulacre (simcult) dans l'espace noyé des médias (mediatrix), en réalisant le rêve post-moderne d'une esthétisation complète de notre société, se déploit dans d'innombrables plis de significations, tant au niveau réel que virtuel. Pour Thomas Mical autant que pour Mark Taylor, la pénétration du cyberspace dans divers corps et diverses organisations humaines (sociales, politiques, physiques, intellectuelles, etc.) non seulement ne fait pas de doute, mais serait aussi propre à déclencher certaines transformations et mutations.

Si ce phénomène de symbiose est souvent abordé,[1] c'est qu'il suggère l'émergence d'une "identité" nouvelle (mi-vivant, mi-machine: espace médiatique). Mais tandis que plusieurs chercheurs en font mention implicitement, d'autres, tel Jim Letwich (Infospace) font carrément le saut. Pour cet informaticien l'Internet est un univers d'intelligences dont la somme produit une conscience planétaire nouvelle.

Enfin je m'en voudrais de ne pas souligner les présentations de Sherry Turkle, de Amy Bruckman-Mitchell Resnick et de Pavel Curtis-David Nichols, car ces trois groupes de chercheurs nous ont proposé d'importantes réfléxions sur le phénomène des MUDs.[2] Peu connu en dehors d'un groupe très ciblé de jeunes utilisateurs, ce phénomène est important car, de par sa structure même, il permet le plongeon dans une méta-fiction organique exclusive à l'Internet (le jeu non seulement sur la fiction propre à chaque MUD, mais aussi sur la fiction psychologique et physiologique de chaque utilisateur). Il représente l'émergence de ce que l'on pourrait appeler la "troisième" oralité, c'est-à-dire une oralité à la fois mythologique et technologique où une exploration d'espaces "sacrés" (psy-chologique, technique, social) s'entreprend au moyen de fictions aux structures "liquides". D'ailleurs, lorsque Amy Bruckman souligne les propriétés psychosociales des MUDs, elle rejoint certaines observations déjà émises par Kérényi sur les effets de miroir et de mise en abyme de la mythologie. 
Ainsi, féconde en chimères architecturales et mythologiques qui offrent aux "plongeurs" explorateurs, coupés des interférences du monde extérieur, un sentiment d'apesanteur et d'immortalité, la structure fractale du cyberspace est essentiellement une matérialisation fluide de l'imaginaire. Car le cyberspace est une typographie qui se constitue comme un palimpseste de langues et de langages (algorithmes, code binaire, pixels, voxels, logiciels, interfaces, etc.), et dont les signes proposent l'existence d'un lieu fascinant, teinté de fantasmes. Dans ses manifestations les plus variées, le cyberspace est bariolé de "surnaturel" construit au moyen de temps, d'espaces et de matérialités autres. Ce lieu dans lequel nous nous conduisons en demi-dieux, en refaisant sans cesse des "gestes originels", possède en fait une structure cosmogonique. Il est un lieu créateur de corps androïdes intelligents, au travers desquels l'être humain et la machine entrent en symbiose. Nous voici confrontés à une carte qui est à elle-même son univers.

Mais il s'agit d'un univers sans référent, puisque le cyberspace n'est qu'un abîme de simulacres [3] où toute frontière disparaît. Car si, dans ce lieu, tout est langues et typographies, alors tout "objet" ou tout "être", nommé et construit par les signes de ces langues, y possède une valeur identique et peut ainsi être échangé ou manipulé à volonté. Les corps présents dans cet espace n'ont aucune hiérarchie. Ainsi, dans cet océan d'absence (absence de référents, absence de réel, absence de physicalité, etc.), il est possible de concevoir qu'un partenariat liquide existe entre tous les éléments qui s'y présentent. Une fois encodée dans le cyberspace, toute organisation devient la parcelle holographique d'un réseau cognitif.

Mais si cette présence est réelle, que représente alors le plongeon dans l'océan cyberspatial? Ou si cet océan est un méta-lieu, un palimpseste vivant, quelles sont alors les conséquences d'une telle présence? $\mathrm{Si}$, de plus, nous sommes à la fois créateurs, spectateurs et les créations de cet "organisme", comment se pose la question de notre interaction avec ce dernier?

Car nous abordons aujourd'hui, à travers l'émergence du cyberspace (ce "quelque chose" qui est à la fois monde-océan, typographie et androïde), la question, à la fois utopique et dystopique, de la mutation des corps (corps physiques, cognitifs et mécaniques). Evidemment, à ce point de jonction, les outils philosophiques que nous possédons deviennent obsolètes puisque de nouvelles associations s'avèrent nécessaires, sinon pour structurer une épistémologie du cyberspace, du moins pour tenter une observation adéquate.

Déjà cet article et cette revue ne sont disponibles qu'à travers l'Internet. Quelle est donc la conséquence du partage, de l'échange ET DE LA SOUMISSION non seulement d'une réflexion, mais aussi d'une typographie, 
à la matérialité cyberspatiale? Sommes-nous, pour emprunter l'expression de Katherine Hayles, entrés dans une époque du post-humain? Et ce "posthumain" est-il enfant de notre civilisation techno-littéraire, résultat de l'hyper-littérarité qui nous forge depuis plusieurs siècles?

Pierre Lévy, Walter Ong et George Landow, parmi d'autres, soulignent avec beaucoup d'à-propos combien déjà androïdes nous sommes par l'intégration psychologique et physique de la "machine" littéraire en nous. Nous pensons, nous communiquons, nous percevons le monde au moyen de cette machine littéraire, profondément imbriquée dans notre fibre cognitive même. Est-il possible alors de postuler que le cyberspace serait cet androïde issu de la présence, dans nos structures cognitives, de la machine littéraire? Et si l'on considère le cyberspace comme un palimpseste liquide et vivant, serait-il concevable de supposer la présence d'un cordon ombilical entre lui et nous? Sommes-nous joints cognitivement au cyberspace? Ressentons-nous le désir profond d'y pénétrer et de nous y noyer parce que ce dernier est le fruit d'une robotisation de l'être humain par la machine littéraire?

Pourrions-nous, enfin, proposer un terme tel que in-humain (plutôt que posthumain) pour définir ce changement? C'est-à-dire que loin d'être un réseau de machines, le cyberspace serait beaucoup plus un réseau de typographies vivantes et ces typographies, le résultat de la lente transformation de l'être humain par les mécanismes et les technologies de la littérarité. Notre corps possède ses propres frontières. La "désincarnation" cyberspatiale ne peut s'accomplir qu'au niveau cognitif, c'est-à-dire, dans notre cas, au niveau littéraire. Lorsque nous pénétrons dans le cyberspace, que ce soit au moyen de la réalité virtuelle ou de l'Internet, lorsque nous nous laissons encoder par son espace-temps, nous ne faisons que plonger en abyme dans notre propre transformation androïde. Le cyberspace est ce méta-lieu construit par la naissance d'une conscience de la machine-littéraire enfouie dans le corps humain. Il annonce un temps et un espace profondément langagier: l'éveil intelligent de l'osmose de l'humain et de la littérarité. L'intelligence artificielle est la première matérialité de cet éveil. Le cyberspace fera probablement émerger les premières réalités de ce monde nouveau.

Nous sommes au seuil d'une profonde révolution. Elle sera, j'ose croire, aussi importante que celle déclenchée par l'imprimerie. Nombre de questions, et surtout nombre d'oppositions (réel/irréel, matériel/immatériel, cognition/computation, etc.) sont réévaluées et surtout repositionnées. La présence de l'ordinateur et de ses réseaux implique, à nos côtés, l'existence de partenaires égaux. Nous sommes à ce point de jonction, qui revient à chaque époque, où la cohérence des perceptions s'altère et mue.[4] Notre structure cognitive, à l'aube d'une ère épistémologique et phénoménologique nouvelle, est aujourd'hui appelée à changer. 


\title{
Ollivier Dyens
}

\author{
Université St. Anne
}

Weynouth, Nova Scotia

Surface Page d'Acceuil/Home Page

[1]Par plusieurs tenant de l'intelligence artificielle mais aussi par des auteurs tels Pierre Lévy, Roger Malina et Gregory Bateson.

[2]Les "Mud"s (Multi-Users Dungeons ou Dimensions), sont des jeux interactifs créés sur l'Internet à partir du modèle "Dungeons and Dragons", c'est-à-dire que tous les participants s'y "habillent" d'une personnalité et d'un sexe quelconque. Ouverts à tous, ces véritables communautés entraînent la création d'une sous-culture complète à l'intérieur même du cyberspace. On peut s'imaginer les MUDs comme une première véritable réalité virtuelle accessible sur l'Internet. Ce qui est intéressant de noter ici est que toute l'interaction, la construction, la modélisation de ces environnements se fait au moyen de l'ECRIT. En entrant dans un des ces jeux une description de l'espace qui vous entoure apparaît à l'écran. Vous interagissez avec cette espace et avec les autres personnages qui l'habitent au moyen de divers commandes et instructions. En fait ces MUDs, à l'architecture souple et profondément créative, sont l'expression de la liquidité du palimpseste cyberspatial.

[3]Le mot "simulacre" n'étant pas ici le plus approprié. Puisque la relation référent-réel/simulacre n'est plus la même dans cet espace-temps, la fonction même du simulacre change elle aussi. Le simulacre devient un réel, puisqu'il renvoie constamment à un autre simulacre. La relation au réel et au référent est coupée.

[4]Pour paraphraser ici Jacob Bronowski. 